\title{
Title:
}

\section{POEMS syndrome: an uncommon cause of peritoneal effusion}

\section{Authors:}

Yuehua Tang, Rencheng Cai, Zhenguo Qiao

DOI: $10.17235 /$ reed.2021.8027/2021

Link: PubMed (Epub ahead of print)

Please cite this article as:

Tang Yuehua, Cai Rencheng, Qiao Zhenguo. POEMS syndrome: an uncommon cause of peritoneal effusion. Rev Esp Enferm Dig 2021. doi: 10.17235/reed.2021.8027/2021.

This is a PDF file of an unedited manuscript that has been accepted for publication. As a service to our customers we are providing this early version of the manuscript. The manuscript will undergo copyediting, typesetting, and review of the resulting proof before it is published in its final form. Please note that during the production process errors may be discovered which could affect the content, and all legal disclaimers that apply to the journal pertain. 
CC 8027

\section{POEMS syndrome: an uncommon cause of peritoneal effusion}

Yuehua Tang ${ }^{1}$, Rencheng $\mathrm{Cai}^{1}$, and Zhenguo Qiao ${ }^{2}$

${ }^{1}$ Department of Gastroenterology. Gaochun People's Hospital. Nanjing, China. ${ }^{2}$ Department of Gastroenterology. Suzhou Ninth People's Hospital. Suzhou Ninth Hospital Affiliated to Soochow University. Suzhou, China

Correspondence: Zhenguo Qiao

e-mail: qzg66666666@163.com

Keywords: POEMS syndrome. Peritoneal effusion. Diagnosis. Treatment.

Conflicts of interest: the authors declare no conflicts of interest.

Dear Editor,

A 46-year-old female was admitted to our hospital with abdominal distension and fatigue of 1 month duration. Physical examination revealed several palpable lymph nodes bilaterally in the axilla and groin, ranging from $0.5 \mathrm{~cm}$ to $2.0 \mathrm{~cm}$ in diameter, with tolerable mobility and no obvious tenderness. Computed tomography (CT) revealed splenomegaly (Fig. 1A, yellow arrow), abdominal effusions (Fig. 1A, blue arrow), and multiple enlarged lymph nodes (Fig. 1B-C, red arrow). To further confirm the diagnosis, the patient underwent a transjugular liver biopsy, and the pathology study ruled out cirrhosis (Fig. 1D). With the progression of her disease, the patient presented with facial erythema, generalized hyperpigmentation, and numbness in both lower limbs. Subsequently, the patient underwent a cervical lymph node biopsy, and the pathology report was consistent with Castleman's disease (Fig. 1E, red arrow). In addition, immunofixation electrophoresis suggested an IgA- $\lambda$ monoclonal protein. Electromyography testing confirmed the presence of impaired nerve conduction velocity in the peroneal, tibial, and sural nerves. Both the clinical presentation and 
testing results suggested POEMS syndrome.

POEMS syndrome is characterized by polyneuropathy $(P)$, organomegaly $(O)$, endocrinopathy (E), monoclonal protein (M), and skin changes (S). The etiology and pathogenesis are still unclear (1). The prevalence rate of POEMS syndrome is about 3 cases per million (2). The patient presented with ascites and splenomegaly, which could easily be misdiagnosed as cirrhosis. However, lymphadenectasis, progressive polyneuropathy, and skin pigmentation provided diagnostic clues for POEMS syndrome. Treatments mainly target the plasma-cell disorder or local lesions (3). Early detection and treatment can significantly improve prognosis in this disease.

\section{REFERENCES}

1. Dispenzieri A, Kyle RA, Lacy MQ, et al. POEMS syndrome: definitions and longterm outcome. Blood 2003;101(7):2496-506. DOI: 10.1182/blood-2002-07-2299

2. Nasu S, Misawa S, Sekiguchi Y, et al. Different neurological and physiological profiles in POEMS syndrome and chronic inflammatory demyelinating polyneuropathy. J Neurol Neurosurg Psychiatry 2012;83(5):476-9. DOI: 10.1136/jnnp-2011-301706

3. Ge $Y, D a Q$, Dai $Y$. Castleman disease of the hyaline vascular variant transforming to POEMS syndrome as endpoint: a case report. BMC Neurol 2018;18(1):169. DOI: 10.1186/s12883-018-1172-7 


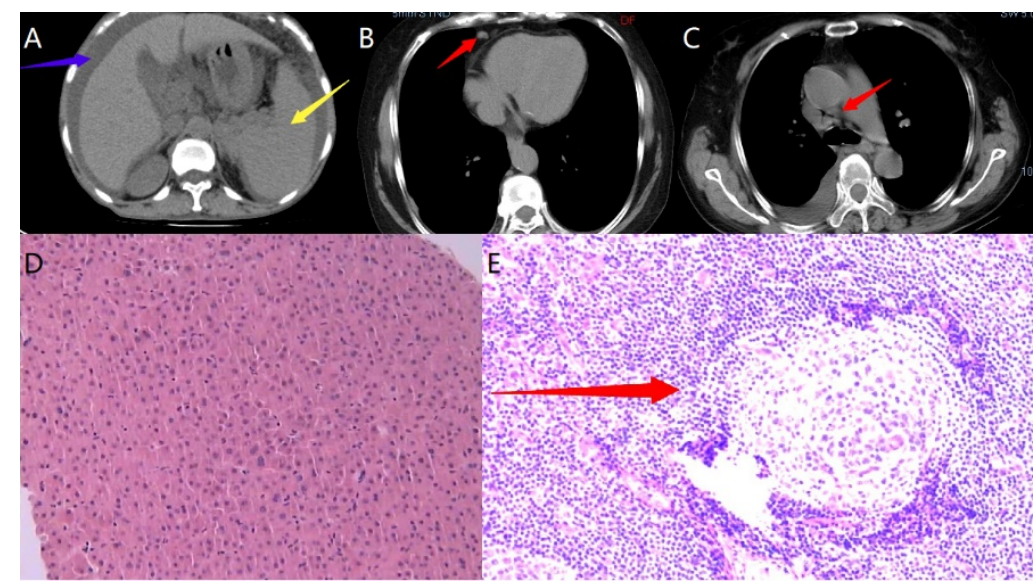

Fig. 1. Computed tomography revealed splenomegaly ( $A$, yellow arrow), abdominal effusions ( $A$, blue arrow), and multiple enlarged lymph nodes (B-C, red arrows). The pathology study of the liver biopsy revealed blood vessels and bile ducts, with no obvious dilatation of the portal area and no obvious infiltration by inflammatory cells. Formation of fibrous partitions was observed in hepatic lobules and the portal area (D). Lymphatic follicles were proliferating and some had an "onion skin" appearance (red arrow), indicating Castleman's disease (E). 